\title{
Optical low-coherence reflectometry with resolution beyond the Fourier transform limit
}

\author{
Chih-Wei Lu, Meng-Tsan Tsai, Yih-Ming Wang, Su-Feng Chen, \\ Yean-Woei Kiang, C.C. Yang * \\ Graduate Institute of Electro-Optical Engineering, Department of Electrical Engineering, National Taiwan University, 1, Roosevelt Road, \\ Section 4, Taipei 106, Taiwan, ROC
}

Received 12 January 2005; received in revised form 29 August 2005; accepted 30 August 2005

\begin{abstract}
We demonstrate experimentally and theoretically a method for enhancing the axial resolution of optical low-coherence reflectometry (OLCR) beyond the Fourier transform limit of light source spectrum. The resolution enhancement originates from the superposition of multiple OLCR scans with different offsets of the center-wavelength light-ray with respect to the rotation axis of the mirror mounted on the galvanometer optical scanner in the used optical phase delay line. After certain software process of the multiple scan fringe patterns of different offsets, the superposition leads to an OLCR axial resolution beyond the transform limit. Experimentally, by using four different offsets for superposition, about 1.5 times enhancement of the transform-limit resolution is obtained.
\end{abstract}

(C) 2005 Elsevier B.V. All rights reserved.

\section{Introduction}

Optical low-coherence reflectometry (OLCR) has been widely used for noninvasive scanning of $\mu \mathrm{m}$-scale structures, particularly in biological tissues [1-4]. It was successfully developed to become a useful technology, named optical coherence tomography (OCT), for medical diagnosis [5]. Basically, this technique uses the interfered signals in a Michelson interferometer for monitoring the backscattered light such that the index-variation structures within a sample can be mapped. The axial resolution cell size of such a system is inversely proportional to the spectral width of the used light source. With a light source of an extremely large spectral width, the axial resolution can be in the range of a few microns [6-12]. The used light sources of extremely large spectral widths include super-luminescence sources [6], mode-locked lasers [8,9], super-continuum generation $[7,10,12]$, and thermal light $[11]$. OLCR or OCT has

\footnotetext{
* Corresponding author. Tel.: +8862 23657624; fax: +886223652637. E-mail address: ccy@cc.ee.ntu.edu.tw (C.C. Yang).
}

caught much attention because of its high-resolution and high-sensitivity scanning capabilities for biomedical diagnosis. Such a high resolution provides us tools for subcellular observations. However, for achieving such a high resolution, costly broadband light sources, such as ultrashort solid-state laser and super-continuum generation pumped with an ultra-short pulse, are needed. The need of high cost and complicated (i.e., unstable) light source systems will eventually hinder the development of compact and inexpensive OLCR or OCT systems for broad applications. Also, in using the light sources of extremely broad bands for achieving high resolution, it becomes difficult to employ the widely used fast-scanning optical phase delay line (OPDL). In this situation, the scanning speed is tremendously reduced [6,9-12]. Meanwhile, with such a broad bandwidth, dispersion compensation becomes a challenging problem, particularly in a fiber-based system. With a free-space OLCR setup, it is usually difficult to implement a compact and stable system.

In this paper, we demonstrate a novel approach for reaching a high resolution with a light source of a relatively small bandwidth in an OLCR system. We can achieve the axial 
resolution beyond the Fourier transform limit of the light source spectrum. The basic idea of implementing such a high OLCR resolution is as follows: we vary the offset, $x_{0}$, of the center-wavelength light-ray with respect to the rotation axis of the mirror, which is mounted on the galvanometer optical scanner (GOS) in the OPDL used in our OLCR system. With different $x_{0}$ values, the multiple longitudinal scans result in different interference fringe patterns. The superposition of the multiple scan images, after certain software process, can lead to a result of a high resolution beyond the Fourier transform limit of the light source spectrum.

This paper is organized as follows: In Section 2, we present the experimental procedures and results. In Section 3, theories behind the experimental implementation are reported. Discussions are given in Section 4. Finally, conclusions are drawn in Section 5.

\section{Experimental procedures and results}

The OLCR system setup used for implementing the highresolution result is shown in Fig. 1. Here, we use a superluminescence diode (SLD) of $950 \mathrm{~nm}$ in central wavelength. After passing through an isolator for protecting the SLD, the effective spectral full-width at half-maximum (FWHM) is $45 \mathrm{~nm}$. Therefore, the theoretical axial resolution is $8.9 \mu \mathrm{m}$ if a Gaussian spectral shape is assumed [13]. In the reference arm, an OPDL is used for group- and phase-delay modulations. To match the optical paths between the reference and sample arms, a fiber length difference of about $25 \mathrm{~cm}$ is required. By using the OPDL and the extra length of dispersive fiber, dispersion of the OLCR system leads to a broadened interference fringe envelope. For changing the $x_{0}$ value, the GOS was placed on a stage translating in the direction perpendicular to the incident optical axis.

In Fig. 2, we show four interference fringe patterns of mirror surface reflection for four $x_{0}$ values, obtained by translating the GOS $1 \mathrm{~mm}$ per step. One can see that the fringes are located at different positions with slightly different widths and chirps. The FWHMs of all the fringe patterns are around $20 \mu \mathrm{m}$. The four fringe patterns in Fig. 2 were processed by (1) shifting the fringes to align the envelope maxima, and

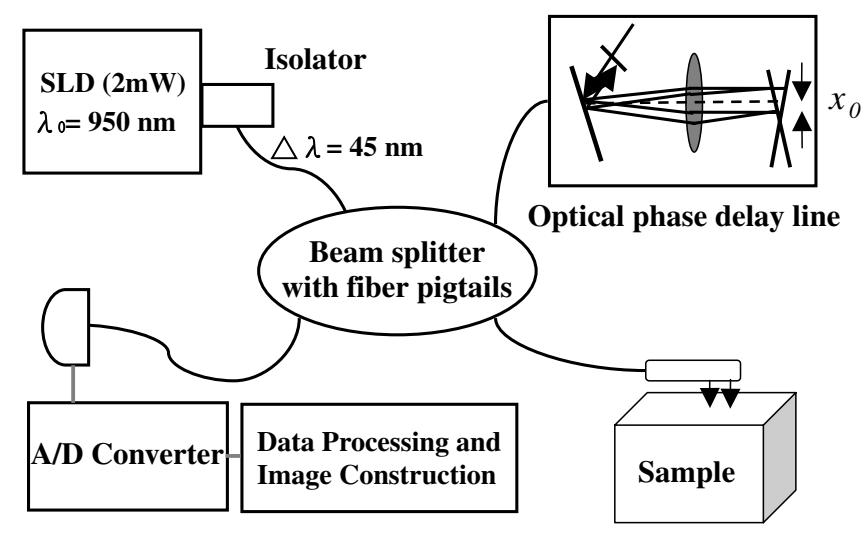

Fig. 1. Experimental setup of the OLCR system.
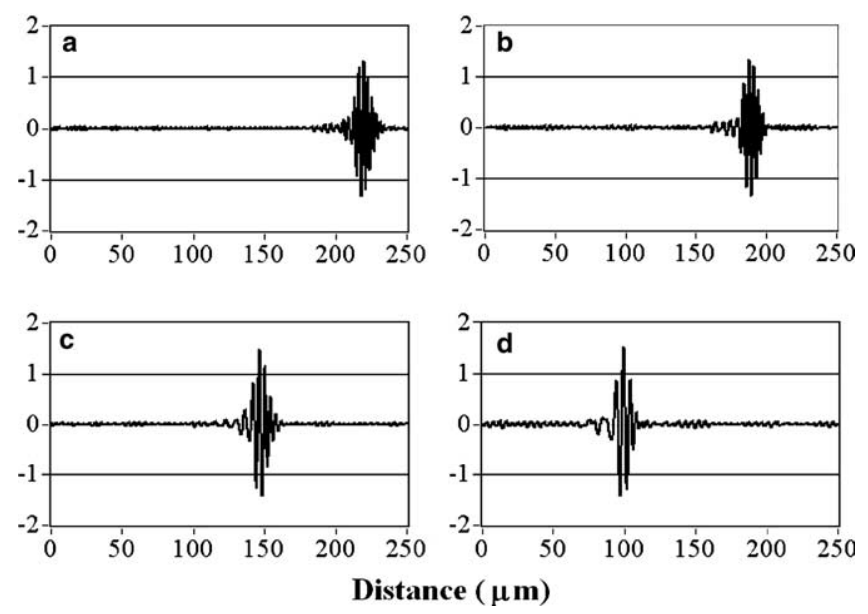

Fig. 2. Four scans of a mirror face with different $x_{0}$ values. The FWHMs are all around $20 \mu \mathrm{m}$.

(2) multiplying the horizontal axis by a factor for each fringe pattern. The multiplication factors are determined by comparing the distances of the fringe envelopes between the two interfaces of a given physical separation in the cases of different $x_{0}$ values. In other words, this multiplication procedure represents the correction of the slight variation in fringe pattern width when different $x_{0}$ values are used. The four multiplication factors for parts (a)-(d) in Fig. 2 are 1, $0.9818,0.9591$, and 0.9321 , respectively. Also, the shifts of the envelope peaks in parts (a)-(d) are 0, 36, 61, and $117 \mu \mathrm{m}$, respectively. The results after the aforementioned software process are shown in Fig. 3. Then, the four fringe patterns in Fig. 3 are superimposed to obtain a new fringe envelope, as shown in Fig. 4. Here, one can see that an OLCR resolution of $5.9 \mu \mathrm{m}$ (FWHM) is achieved. This value is about two-third the Fourier transform limit.

To further demonstrate the resolution enhancement, we scanned a sample consisting of two contacting glass plates with the aforementioned procedure. Parts (a)-(c) in Fig. 5
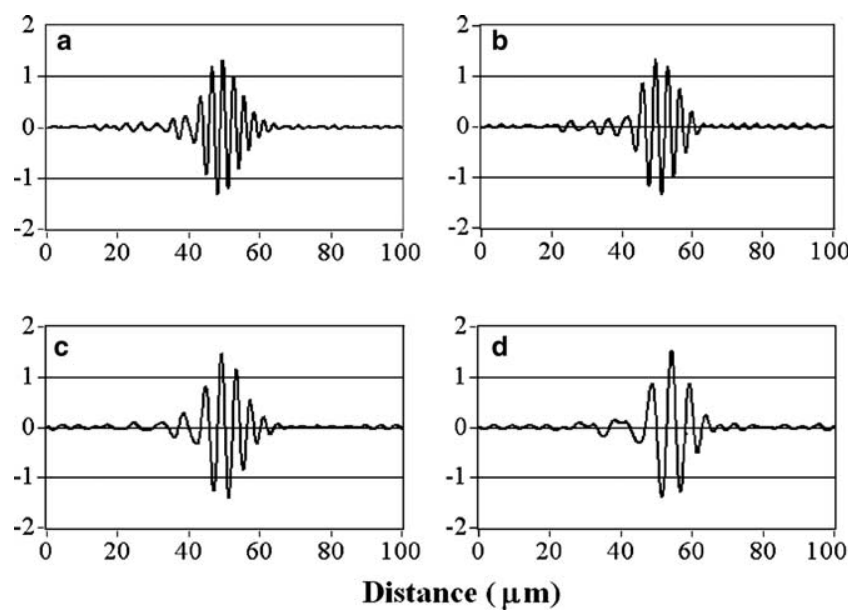

Fig. 3. The fringe patterns of the four scanning results in Fig. 2 after the software process. 


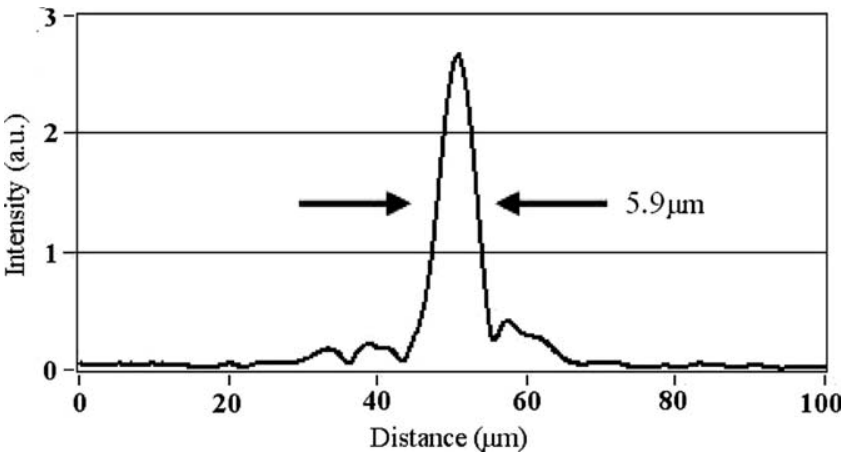

Fig. 4. The fringe envelope after the superposition of the four fringe patterns in Fig. 3. The OLCR axial resolution becomes $5.9 \mu \mathrm{m}$.

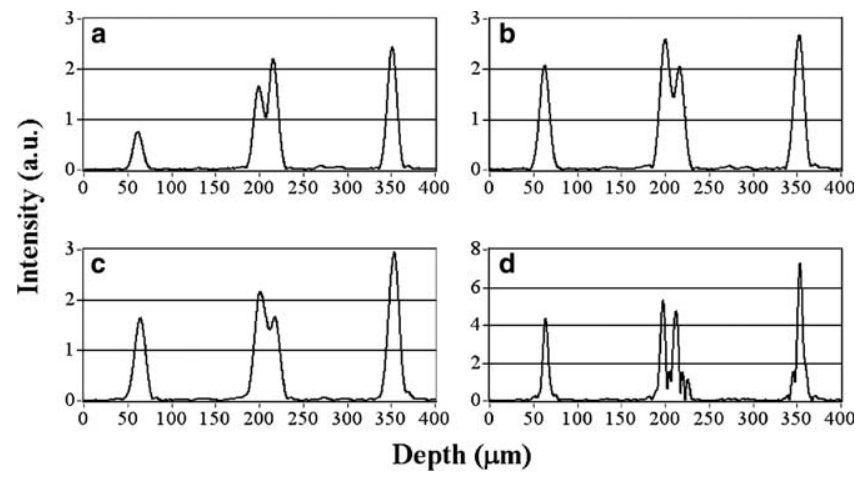

Fig. 5. (a)-(c) 1-D scanning results of a sample consisting of two contacting glass plates with different $x_{0}$ values. (d) Superposition result of parts (a)-(c) after the software process.

shows the 1-D scanning results with three different $x_{0}$ values. Here, although the nearby interfaces between the two glass plates can be more or less identified in parts (a)-(c), their separation is much better resolved in part (d), which is obtained by combining parts (a)-(c) following the procedures described above. The resolution in part (d) is clearly enhanced.

\section{Theories}

To understand the theories behind the resolution enhancement, we demonstrate the concept with the following derivations. For the convenience in derivation, we use the frequency and time domains $(\omega, t)$, instead of the domains of wave number and space. Their correspondence is quite straightforward. The phase evolution, $\phi(\omega, t)$, of an optical signal after returning from a typical OPDL can be expressed as [14]

$$
\begin{aligned}
\phi(\omega, t) & =\frac{8 \pi \cdot f \cdot \Gamma t}{\lambda}\left(\theta_{0}-\sin ^{-1}\left(\frac{\lambda}{d}-\sin \theta_{i}\right)+\frac{x_{0}}{f}\right) \\
& \approx \phi\left(\omega_{0}, t\right)+\left.\frac{\partial \phi}{\partial \omega}\right|_{\omega=\omega_{0}}\left(\omega-\omega_{0}\right) .
\end{aligned}
$$

Here, $\omega$ is the angular frequency, $\lambda$ is the wavelength, $f$ is the focal length of the used lens, $\Gamma$ is the rotation angular frequency of the GOS, $d$ is the grating period, $\theta_{0}$ is the incident angle onto the diffractive grating, and $\theta_{1}$ is the diffracted angle from the grating. In (1), we series-expand the phase $\phi(\omega, t)$ and keep only the zeroth and first-order terms. The higher-order terms, which are related to dispersion, are ignored in our concept demonstration. Here, $\omega_{0}$ is the central angular frequency. For expression simplicity, we define $\alpha\left(x_{0}\right)$ and $\beta\left(x_{0}\right)$ as

$$
\begin{aligned}
& \phi\left(\omega_{0}, t\right)=\frac{8 \pi \Gamma x_{0}}{\lambda_{0}} t=\alpha\left(x_{0}\right) \cdot t, \\
& \left.\frac{\partial \phi}{\partial \omega}\right|_{\omega=\omega_{0}}=\beta\left(x_{0}\right) \cdot t .
\end{aligned}
$$

Note that $\alpha=0$ when $x_{0}$ is zero. In collecting the interfered signals, we are concerned with the cross-correlation function, $C_{r}(t)$, between the reference and sample arms as

$$
\begin{aligned}
C_{r}(t)= & \int\left\langle\tilde{E}_{s}\left(t^{\prime}\right) \tilde{E}_{r}^{*}\left(t^{\prime}+t\right)\right\rangle \mathrm{d} t^{\prime} \\
= & \frac{E_{0}^{2}}{2 \pi} \exp \left[-\mathrm{j}\left(\alpha+\omega_{0}\right) t+\mathrm{j} \omega_{0} \tau_{g s}\right] \\
& \times \int G\left(\omega^{\prime \prime}\right) \exp \left[\mathrm{j}\left(\tau_{g s}-(1+\beta) t\right) \omega^{\prime \prime}\right] \mathrm{d} \omega^{\prime \prime} \\
= & E_{0}^{2} \exp \left[-\mathrm{j}\left(\alpha+\omega_{0}\right) t+\mathrm{j} \omega_{0} \tau_{g s}\right] \cdot \tilde{G}\left(\tau_{g s}-(1+\beta) t\right) .
\end{aligned}
$$

Here, $\tilde{E}_{s}\left(t^{\prime}\right)$ and $\tilde{E}_{r}^{*}\left(t^{\prime}+t\right)$ are the time-domain optical signals returned from the sample and reference arms, respectively, and $E_{0}$ is their equal amplitude. The symbol $\langle\bullet\rangle$ represents the ensemble average. Also, $\tau_{g s}$ stands for the group delay difference between the two arms. It is also a function of $x_{0}$. Meanwhile, $G(\omega)$ is the light source spectral intensity and $\tilde{G}(t)$ is the counterpart in the time domain. From (4), one can see that a nonzero $x_{0}$ (nonzero $\alpha$ ) leads to the shift of the modulation frequency of the interference fringe pattern. Also, the $\beta$ factor results in the modification of the time axis and the variation of fringe envelope peak position. The $x_{0}$ dependence of $\tau_{g s}$ also contributes to the peak position variation of fringe envelope.

To show the resolution enhancement through the superposition of more than one scanning results of different $x_{0}$ values, we consider an example of two $x_{0}$ values as follows:

$$
\begin{aligned}
& C_{r 1}(t)=E_{0}^{2} \exp \left[-\mathrm{j}\left(\alpha_{1}+\omega_{0}\right) t+\mathrm{j} \omega_{0} \tau_{g s 1}\right] \cdot \tilde{G}\left(\tau_{g s 1}-\left(1+\beta_{1}\right) t\right), \\
& C_{r 2}(t)=E_{0}^{2} \exp \left[-\mathrm{j}\left(\alpha_{2}+\omega_{0}\right) t+\mathrm{j} \omega_{0} \tau_{g s 2}\right] \cdot \tilde{G}\left(\tau_{g s 2}-\left(1+\beta_{2}\right) t\right) .
\end{aligned}
$$

The software process described in Section 2 implies the modification of (6) as

$$
\begin{aligned}
C_{r 2}^{\prime}(t)= & E_{0}^{2} \exp \left[-\mathrm{j}\left(\alpha_{2}+\omega_{0}\right) \frac{\left(1+\beta_{1}\right)}{\left(1+\beta_{2}\right)} t+\mathrm{j} \omega_{0} \tau_{g s 1}\right] \\
& \cdot \tilde{G}\left(\tau_{g s 1}-\left(1+\beta_{2}\right) \frac{\left(1+\beta_{1}\right)}{\left(1+\beta_{2}\right)} t\right) \\
= & E_{0}^{2} \exp \left[-\mathrm{j} a\left(\alpha_{1}+\omega_{0}\right) t+\mathrm{j} \omega_{0} \tau_{g s 1}\right] \\
& \cdot \tilde{G}\left(\tau_{g s 1}-\left(1+\beta_{1}\right) t\right) .
\end{aligned}
$$




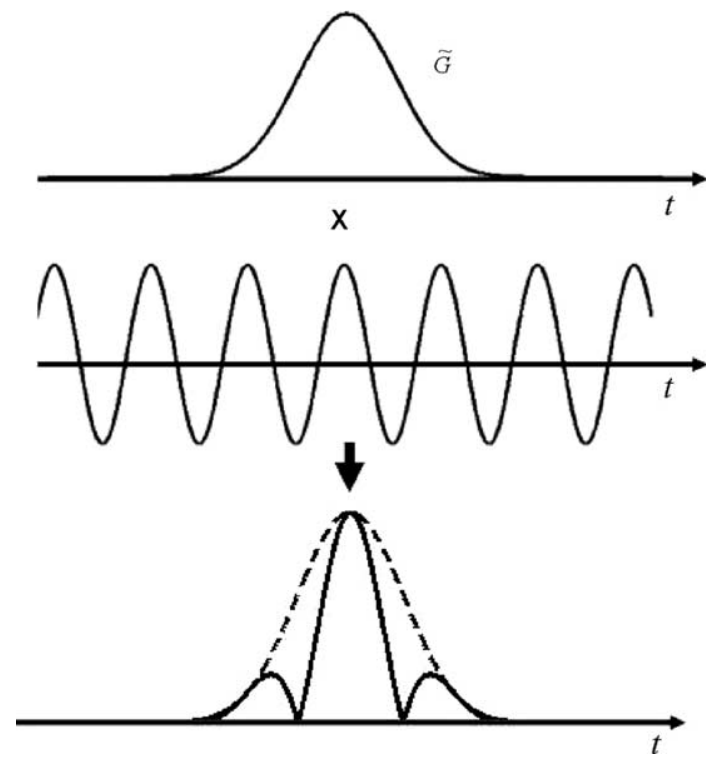

Fig. 6. Schematic demonstration of the OLCR resolution modification to reach a level beyond the Fourier-transform limit.

In other words, the time scale is modified by a factor of $\left(1+\beta_{1}\right) /\left(1+\beta_{2}\right)$. Also, the peak position of the fringe envelope is shifted by $\tau_{g s 1}-\tau_{g s 2}$. Here,

$a=\frac{\left(\alpha_{2}+\omega_{0}\right)\left(1+\beta_{1}\right)}{\left(\alpha_{1}+\omega_{0}\right)\left(1+\beta_{2}\right)}$.

Therefore, the superposition leads to

$$
\begin{aligned}
C_{r}^{(2)}(t)= & C_{r 1}(t)+C_{r 2}^{\prime}(t) \\
= & E_{0}^{2} \exp \left(\mathrm{j} \omega_{0} \tau_{g s 1}\right)\left\{\exp \left[-\mathrm{j}\left(\alpha_{1}+\omega_{0}\right) t\right]\right. \\
& \left.+\exp \left[-\mathrm{j} a\left(\alpha_{1}+\omega_{0}\right) t\right]\right\} \cdot \tilde{G}\left(\tau_{g s}-\left(1+\beta_{1}\right) t\right) .
\end{aligned}
$$

The two terms in the curved brackets produce beating to form a sinusoidal modulation for the fringe envelope, as schematically shown in Fig. 6. Here, side-lobes can be seen in the final fringe envelope (after taking the absolute value). Such a theoretical result may explain the side-lobes in Fig. 4. The side-lobes can be reduced if more scans of different $x_{0}$ values are considered. Theoretically, if the $x_{0}$ values can be in multiple, the superposition of these scan components is similar to the case of laser mode locking, in which coherent frequency components are superimposed to form a short pulse. Therefore, a narrow hump can be produced for modifying the fringe envelope, leading to an OLCR resolution beyond the Fourier transform limit of the source spectrum.

\section{Discussions}

Resolution beyond the transform limit in an OLCR system is useful for building high-resolution systems with low-cost light sources. However, the proposed approach requires multiple scans of a sample that may require a longer imaging time. When the GOS is operated at $1 \mathrm{kHz}$, the required imaging time for a four-scan experiment includes:
(1) $<0.4 \mathrm{~s}$ for the four scans of a $1 \mathrm{~mm} \times 1 \mathrm{~mm}$ frame (100 A-mode scans per frame); (2) $3 \mathrm{~s}$ for changing the $x_{0}$ value; and (3) $<0.8 \mathrm{~s}$ for data process. Hence, the four-scan experiment requires $<4.2 \mathrm{~s}$, which is about 40 times that of single-scan imaging. Nevertheless, the requirement of such a multiple-scan operation with a light source of a relatively narrower bandwidth does not necessarily represent a drawback in an OLCR or OCT system when compared with the case of an extremely broad bandwidth. With a light source of an extremely broad bandwidth, the fast-scanning OPDL can no longer be used in such a system due to the limited width of the mirror mounted on the GOS. The use of other axial scanning mechanisms, such as a constant-speed translation stage, makes the scanning speed thousand times slower than that using an OPDL [6,9-12]. For instance, with $1 \mathrm{~Hz}$ operation frequency of a translation stage, a $1 \mathrm{~mm} \times 1 \mathrm{~mm}$ image requires $100 \mathrm{~s}$, which is longer than that of the aforementioned four-scan experiment. Therefore, if the multiple-scan method with a reasonably narrow source bandwidth can lead to an OLCR or OCT resolution comparable to that of a system with an extremely large spectral width, the multiple-scan does not necessarily represent a drawback.

Regarding the imaging time, another important point deserves further discussions as follows. In operating an OLCR or OCT system with various offsets, $x_{0}$, we do not need to adjust the $x_{0}$ values or search for the matching conditions by calculating $\alpha, \beta$, and $\tau_{g s}$ for an individual sample. We simply need to choose one set of $x_{0}$ values and optimize the process parameters in advance. Once those parameters are optimized, they can be used for scanning various samples. Therefore, the key problem in imaging time elongation in the proposed method is the change of the $x_{0}$ value. The variation of the $x_{0}$ value for multiple scans is indeed impractical. By using a phase modulator for multiplefrequency scanning will be practical. In this situation, only a few milliseconds is needed for changing the modulation frequency. Recently, the development of spectral-domain OCT has led to much higher scanning speed, up to tens frames per second, which is beyond the video rate [15]. With this development, the required longer imaging time due to the multiple-scan procedure should not be a major problem.

The compensation of the system dispersion mismatch is a crucial issue in the operation of an OLCR or OCT system. In our derivations, we considered only up to the term of the first-order derivative with respect to frequency in (1) for concept demonstration. If we considered the term of the second-order derivative (dispersion effect), Eq. (4) would become more complicated. In this situation, the data process procedures for the superposition of multiple-scan results might become difficult. Also, the implementation of a resolution beyond the transform limit is ineffective. A significant dispersion effect will broaden the fringe envelope and hence cancel the resolution improvement. Therefore, our idea for improving the resolution is designed for an OLCR or OCT system with a relatively smaller spectral 
width. In such a system, the dispersion can be easily compensated.

Sample material dispersion is a key issue in evaluating the function of the proposed method even the system spectral width is not broad. In this situation, the conditions for resolution improvement can still be optimized through a careful process of the data, i.e., adjusting the required parameters. After such an optimization procedure, the OLCR or OCT system can be used for scanning all samples of the similar material dispersion property. In other words, the system parameters do not need frequent adjustments. Finally, the major effect of scattering in tissues is to attenuate the returned signal intensity. Scattering actually does not have the direct impact on the proposed method.

\section{Conclusions}

In summary, we have demonstrated experimentally and theoretically a method for enhancing the axial resolution of an OLCR or OCT system beyond the Fourier transform limit of light source spectrum. The resolution enhancement originated from the superposition of multiple longitudinal scans with different offsets of the centerwavelength light-ray with respect to the rotation axis of the mirror mounted on the GOS in the used OPDL. After certain software process of the multiply scanned fringe patterns of different offsets, the superposition led to a resolution beyond the Fourier transform limit. Experimentally, by using four different offsets for superposition, about 1.5 times enhancement of the transform-limit value was obtained.

\section{Acknowledgment}

This research was supported by National Health Research Institute, The Republic of China, under the Grant of NHRI-EX94-9220EI.

\section{References}

[1] X. Clivaz, F. Marquis-Weible, R.P. Salathe, R.P. Novak, H.H. Gilgen, Optics Letters 17 (1992) 4.

[2] X.J. Wang, T.E. Milner, R.P. Dhond, W.V. Sorin, S.A. Newton, J.S. Nelson, Optics Letters 20 (1995) 524.

[3] U. Wiedmann, P. Gallion, G.H. Duan, Journal of Lightwave Technology 16 (1998) 1343.

[4] Y.L. Kim, J.T. Walsh Jr., M.R. Glucksberg, Applied Optics 42 (2003) 6959.

[5] D. Huang, E.A. Swanson, C.P. Lin, J.S. Schuman, W.G. Stinson, W. Chang, M.R. Hee, T. Flotte, K. Gregory, C.A. Puliafito, J.G. Fujimoto, Science 254 (1991) 1178.

[6] A.M. Kowalevicz, T. Ko, I. Hartl, J.G. Fujimoto, Optics Express 10 (2002) 349.

[7] D.L. Marks, A.L. Oldenburg, J.J. Reynolds, Optics Letters 27 (2002) 2010.

[8] A. Unterhuber, B. Považay, B. Hermann, H. Sattmann, W. Drexler, Optics Letters 28 (2003) 905.

[9] K. Bizheva, B. Považay, B. Hermann, H. Sattmann, W. Drexler, Optics Letters 28 (2003) 707.

[10] B. Povazay, K. Bizheva, A. Unterhuber, B. Hermann, H. Sattmann, A.F. Fercher, W. Drexler, Optics Letters 27 (2002) 1800.

[11] L. Vabre, A. Dubois, A.C. Boccara, Optics Letters 27 (2002) 530.

[12] Y. Wang, Y. Zhao, J.S. Nelson, Z. Chen, Optics Letters 28 (2003) 182.

[13] F. Lexer, C.K. Hitzenberger, A.F. Fercher, M. Kulhavy, Applied Optics 36 (1997) 6548.

[14] G.J. Tearney, B.E. Bouma, J.G. Fujimoto, Optics Letters 22(1997) 1811.

[15] A.F. Fercher, C.K. Hitzenberger, G. Kamp, S.Y. Elzaiat, Optics Communications 117 (1995) 43. 